\title{
The Spurious-free Dynamic Range Measurement for RF Receiving System
}

\author{
Rui Jin ${ }^{a}$, Zhiyong Yu, Wenxiang Guo \\ Xi'an Hong Qing High Tech Research Institute, Xi'an, China \\ a2231118252@qq.com
}

\begin{abstract}
In this paper, the upper and lower limits measurement method of the spurious-free dynamic range is proposed. With analyzing the error source in measurement, the error correction is given for the upper limit measurement, and improved the lower limit measurement method.
\end{abstract}

Keywords: the spurious-free dynamic range, RF receiving system, error analysis.

\section{Introduction}

It is an important index to measure the the spurious-free dynamic range of RF receiving system.

In the spurious-free dynamic range, the upper limit value is the sensitivity level of the system and the upper limit is IIP3 of the system The measurement method of the spurious-free dynamic range were analyzed in reference [1].the error sources of the spurious-free dynamic range measurement were analyzed in reference [2-4].In these studies, they lack the quantitative analysis of error in the upper and lower limits measurement. This paper focuses on the spurious-free dynamic range measurement for RF receiving system, giving the error correction to the upper limit measurement and improved the lower limit measurement method.

\section{The Lower Limit of the Spurious-Free Dynamic Range}

\subsection{Basic Principles}

To measure the internal noise performance of a complex system, the concept of noise figure was introduced and defined as:

$$
F=\frac{S_{i} / N_{i}}{S_{o} / N_{o}}
$$

Among them, $S_{i}$ is input signal power; $N_{i}$ is input noise power; $S_{o}$ is output signal power; $N_{o}$ is output noise power.

It can be seen from formula (1) that noise figure is a parameter that does not depend on the sampling bandwidth of the system. Therefore, noise figure is the most important standard parameter to measure the noise performance of receiver system. After considering the noise floor and noise figure of the system, the overall noise power or level value of the receiver system can be obtained. The minimum input signal level value to ensure the normal operation of the receiver system must be higher than the value. There are many kinds of sensitivity according to the different uses and requirements of the receiver. The application mode of the monitoring receiver and the communication receiver used in the spectrum monitoring is different. the lower limit of the receiver system's false response dynamic range can be defined as the minimum signal detection sensitivity (Minimum Detectable Signal), which is referred to as MDS, and the calculation formula is as follows:

$$
M D S=-174 \mathrm{dBm} / \mathrm{Hz}+N F+10 \lg \left(\frac{R B W}{1 \mathrm{~Hz}}\right)+3 \mathrm{~dB}
$$

Where: RBW refers to the system resolution bandwidth, $3 \mathrm{~dB}$ is ITU-R recommended correction value. When measuring the performance of the receiver system, the lower the noise figure and the MDS value, the better the noise performance. It can also be regarded as the lower limit of its dynamic range. The variable in formula (2) is the noise figure and the resolution bandwidth, and the noise figure is proportional to the system gain, so the bandwidth and gain of the system are the main parameters affecting the MDS. In the spectrum monitoring, if the signal of small field intensity is to 
be measured, the system sensitivity can be improved by changing the above parameters to ensure that the spectrum data of small signals are not lost.

\subsection{Measurement}

The RF receiving system generally consists of a receiving antenna, RF cables, an amplifier and a receiver. In the process of measuring the noise figure of the RF receiving system using the Y-factor method, the receiver of the RF receiving system can be directly used as a measuring instrument to realize the measurement of the noise figure of the RF receiving system. The principle is shown in Figure 1. Among them, the equivalent noise temperature $(\mathrm{K})$ when the normal temperature load (50 $\Omega), T_{e 1}$ is the equivalent noise temperature $(\mathrm{K})$ at the antenna end, $T_{e 2}$ is the equivalent noise temperature $(\mathrm{K})$ of the rear amplifier chain, and $T_{e}$ is the equivalent noise of the RF receiving system Temperature $(\mathrm{K})$.

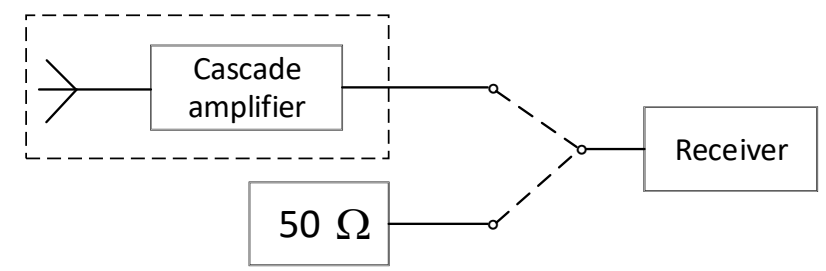

Figure 1. Principle of system noise figure measurement

Adjusting the antenna to a clean electromagnetic environment. At this time, the noise power $N_{e}$ measured by the spectrum analyzer is:

$$
N_{e}=k\left(T_{e 1}+T_{e 2}\right) B G_{e 2}
$$

Among them, $N_{e}$ is the noise power of the RF receiving system, $G_{e 2}$ is the equivalent gain of the. The normal temperature load is directly connected to the spectrum analyzer, and the measured noise power $N_{\text {noise }}$ is:

$$
N_{\text {noise }}=k T_{o} B
$$

Dividing formula (4) by formula (3), and expressing the ratio by Y-factor:

$$
\mathrm{Y}=\frac{N_{\text {noise }}}{N_{e}}=\frac{T_{o}}{\left(T_{e 1}+T_{e 2}\right) G_{e 2}}
$$

In general, $G_{e 2}$ is the gain of the post stage amplifier chain, the system noise temperature can be expressed as:

$$
T_{e}=T_{e 1}+T_{e 2}=\frac{T_{o}}{G_{e 2} Y}
$$

The system noise figure is calculated as:

$$
F=1+\frac{1}{G_{e 2} Y}
$$

\subsection{Error Analysis and Correction}

The Y-factor is the core of the Y-factor method to measure the noise figure of the system. Due to the influence of the noise floor of the receiver, the error of the measured noise power appears, which causes the error of the $\mathrm{Y}$-factor and causes the error of the measurement of the system noise figure,

Setting the receiver noise floor to $N_{\text {floor }}[\mathrm{dBm}]$, the measured noise power to $N_{\text {mea }}[\mathrm{dBm}]$, and the actual noise power to $N_{a c t}[\mathrm{dBm}]$. Then the measured noise power $N R_{\text {mea }}$ and actual noise power $N R_{a c t}$ are:

$$
\begin{gathered}
N R_{\text {mea }}=N_{\text {mea }}-N_{\text {floor }} \\
N R_{\text {act }}=N_{\text {act }}-N_{\text {floor }}
\end{gathered}
$$


The relationship between the measurement noise power ratio and the actual noise power ratio is:

$$
N R_{\text {act }}=10 \times \lg \left(10^{\frac{N R_{\text {mea }}}{10}}-1\right)
$$

The correction factor of noise power measurement $\delta_{n}$ is defined as:

$$
\delta_{n}=N R_{\text {mea }}-N R_{\text {act }}=N R_{\text {mea }}-10 \times \lg \left(10^{\frac{N R_{\text {mea }}}{10}}-1\right)
$$

Setting the correction factors for the system noise power and the load noise power are $\delta_{e}$ and $\delta_{\text {noise }}$, the actual power sums of the two measurements are $N_{e_{-} \text {act }}$ and $N_{\text {noise_act }}$ :

$$
\begin{aligned}
N_{e_{-} a c t} & =N_{e}-\delta_{e} \\
N_{\text {noise_act }} & =N_{\text {noise }}-\delta_{\text {noise }}
\end{aligned}
$$

The system noise figure is calculated as:

$$
F=1+\frac{\left(N_{e}-\delta_{e}\right)}{G_{e 2}\left(N_{\text {noise }}-\delta_{\text {noise }}\right)}
$$

\section{The Upper Limit of the Spurious-Free Dynamic Range}

\subsection{Basic Principles}

When the input signal level increases to the saturation level of the electronic components, the receiver system enters the nonlinear working area and produces output distortion. There will be the output distortion of intermodulation products and reciprocal frequency mixing, in which the intermodulation products have the most serious influence on the spectrum monitoring. When the intermodulation product level value is greater than the minimum signal detection sensitivity, the receiving chance gives a false signal response. In the multi signal input receiver system, the two order cut-off point (IP2) and the three order cut-off point (IP3) are commonly used to characterize the linearity of the receiver system.

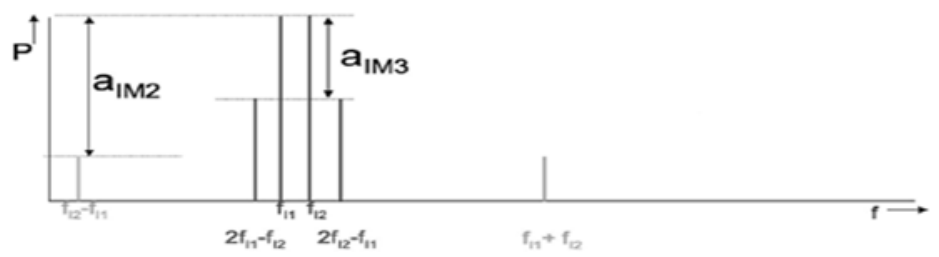

Figure 2. Intermodulation Product Schematic

As shown in Figure 2, $f_{11}$ and $f_{12}$ are the input signal frequency, $f_{12}-f_{11}$ and $f_{11}+f_{12}$ are the two order intermodulation products, $2 f_{11}-f_{12}$ and $2 f_{12}-f_{11}$ are the three order intermodulation products, $\mathrm{a}_{I M 2}$ and $\mathrm{a}_{I M 3}$ are the order intermodulation products are worth the difference with the input signal level. The formulas for IP2 and IP3 are as follows:

$$
\begin{aligned}
& I P 2=P_{\text {in }}+\mathrm{a}_{I M 2} \\
& I P 3=P_{\text {in }}+\mathrm{a}_{I M 3} / 2
\end{aligned}
$$

It can be seen from Figure 2,the frequency of the second-order and third-order intermodulation products is linearly related to the input frequency and appears at the sum or difference of the two input frequencies or the difference between them. In the case where the system IP2 and IP3 have been determined, the larger the input signal, the larger the intermodulation product level value. The above formula and theory also apply when multiple signals enter the receiver system's passband at the same time. When the input receiver system signal level value increases to a certain value, the second-order third-order intermodulation product level value calculated according to IP2 and IP3 will exceed the minimum signal detection sensitivity level, causing in a false response of the receiver. The maximum input signal level that causes a false response is the upper limit of the dynamic range without false response, and the difference between the minimum signal detection sensitivity level and the 
maximum input signal level value that does not generate a false response is a false response dynamic range. According to the ITU-R Recommendations, the receiver system has a spurious free dynamic range (SFDR) calculation formula:

$$
\begin{aligned}
S F D R 2 & =\frac{1}{2}(I P 2+174-N F-10 \lg R B W) \\
S F D R 3 & =\frac{2}{3}(I P 3+174-N F-10 \lg R B W)
\end{aligned}
$$

According to equations (17) and (18), it can be seen that the key parameters affecting the receiver system's spurious-free response dynamic range are the noise figure IIP2 and IIP3 intercept point value. These two parameters can be used to measure the dynamic range of the receiver system without false response. Changing the value can also adjust the upper and lower limits of the system dynamic range.

\subsection{Measurement}

The second-order, third-order intermodulation intercept points reflect the receiver's ability to process signals linearly. The intersection point where the second-order third-order intermodulation product output of the receiver increases with the input signal and the fundamental wave output with the extension of the input signal increases is called receiver second-order third-order intermodulation intercept point, and the unit of measurement is $\mathrm{dBm}$. The output second-order intermodulation intercept point power level is referred to as the output second-order intermodulation intercept point; the output third-order intermodulation intercept point power level is referred to as the output thirdorder intermodulation intercept point; the output intercept point power level is converted to the input At the end of the time, it is called input second-order third-order intermodulation intercept power level.

The measurement of the intermodulation intercept point value is a complicated matter. It requires two annunciators to generate the interference signal and is fed to the receiver through a combiner at the same time. In the test, we must first ensure that the test system itself will not bring error, especially in high-linear devices. Just as using a spectrum analyzer to measure the noise figure of an amplifier or using a source analyzer to measure the phase noise of a frequency synthesizer, make sure that the noise figure of the spectrum analyzer itself or the phase noise of the source analyzer is better than the device under test ( DUT) An order of magnitude or more to ensure the accuracy of the measurement.

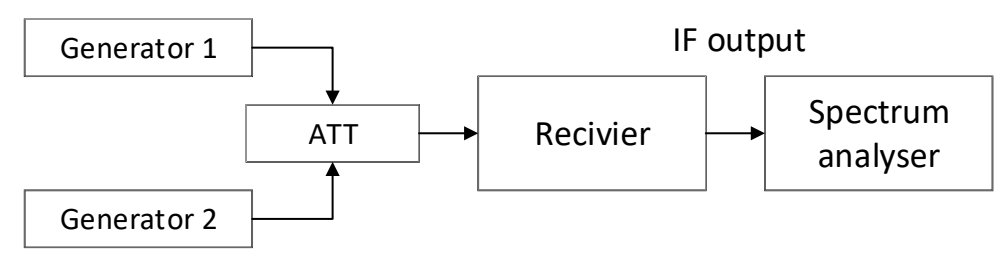

Figure 3. Mutual adjustment point test

The measurement procedure is as follows:

a) Connecting the measurement system as shown in the figure. 3

b) The receiver under test is set at the operating frequency FS;

c) The signal generator 1 outputs a single-carrier test signal with a frequency of $F_{s}+\Delta F$, and the output power is $\mathrm{Pi}$. The signal generator 2 outputs a single-carrier test signal with a frequency of $2 F_{s}+\Delta F$, and the output power is also set to Pi. The selection should be such that the output frequencies of signal generators 1 and 2 are in the same preselect;

d) Observing the receiver IF output, and the measurement equipment records the receiver's IF output power P2;

e) Turning off the signal generator 2 and adjust the output frequency of the signal generator 1 to Fs;

f)adjusting the output power of the signal generator 1 so that the receiver IF output power is also $\mathrm{P} 2$, record the output power of the signal generator 1 is $P_{i}^{\prime}$, at this time, calculate the signal generator 1 output power variation is $\Delta P_{i}=P_{i}-P_{i}^{\prime}$; 
g) Calculating the receiver's input second-order intermodulation intercept point IP2 $=\mathrm{Pi}-$ the combiner attenuation $+\Delta \mathrm{Pi}$.

h) Changing the operating frequency FS and repeat steps b) to g) to obtain the second-order intermodulation intercepts measured at different frequencies.

\subsection{Error Analysis and Improvement}

The conventional intermodulation intercept point value testing system only synthesizes two channels of signal irrigation to the DUT. The factors that may cause the test error are:

(1) The isolation between the signal sources is not high, causing crosstalk between the signal sources.

(2) The dynamic range of the spectrum analyzer itself is only about $80 \mathrm{~dB}$, which will produce distortion products.

(3) Passing intermodulation caused by-factors such as RF cables, combiners and even radio frequency heads in the test system.

Therefore, for highly linear devices, the nonlinearity of the test system itself is required to be at least $15 \mathrm{~dB}$ higher than the DUT. A suitable test system is shown in Figure 4. After the signal is amplified and attenuated (the total gain of the two is $0 \mathrm{~dB}$ ) and then combined, the isolation between the signal devices can be improved. The role of the low-pass filter is to reducing the intermodulation products, so that the interference signals are relatively pure. DUT, the role of high-pass filter is to filter out the interference signal, only let the intermodulation product display on the spectrum analyzer, reducing the impact of spectrum analyzer distortion.

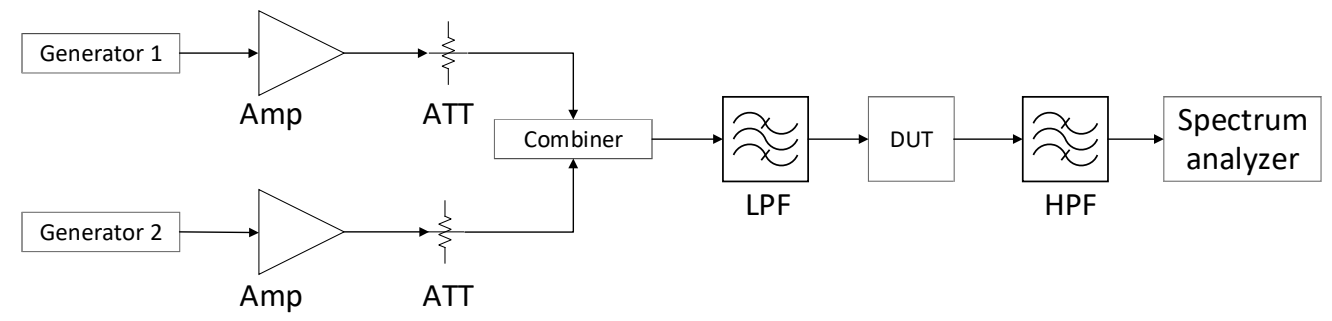

Figure 4. Intermodulation intercepts to improve measurement

\section{Conclusion}

The spurious-free dynamic range of the RF receiver system can have a significant impact on the accuracy of the spectrum monitoring data. In this paper, the upper and lower limits measurement method of the spurious-free dynamic range is proposed. With analyzing the error source in measurement, the error correction is given for the upper limit measurement, and improved the lower limit measurement method. It has a good influence to the monitoring work.

\section{References}

[1]. Nellie Pang,Peter Kronseder,ReceiverParameters,ROHDE\&SCHWARZ.

[2]. Nellie Pang,Peter Kronseder,DSP in Receiver. ROHDE\&SCHWARZ.

[3]. Kim M Harrington. Active Array Nonlinearity Requirements-Special Analysis of Third Order Inter-modulation Clutter. IEEE Transactions on Phased Array Technology, 1996.

[4]. D.C.Schleher. MTI and Pulsed Doppler Radar. Artech House. Boston, London, 1991.

[5]. G.L.Herter. Characterization of Nonlinearities in Microwave Devices and Systems. IEEE Trans. M.T.T,Dec. 1973.

[6]. J.Eaves and E.Reed, eds. Principles of Modern Radar. Van Nstrand Reinhold, New York,1987.

[7]. MORITA R Y. Psychrophilic bacteria[J]. Bacterial Rev,1975, 39:146-167. 
[8]. CHAURET CY, Mayfield C I. Biotransformation of dinbutyl phthalate by a psychrotrophic Pseudomonas fluorescens(BGW) isolated from subsurface environment[J]. Can JMicrobiol, 1995,41:54-63.

[9]. JARVINEN K T, Melin E S. High-rate bioremediation of chlorophenol contaminated groundwater at low temperatures[J]. Environ Sci Technol, 1994,28:2387-2392. 